\title{
Ceramide induces the apoptosis of non-small cell lung cancer cells through the Txnip/Trx1 complex
}

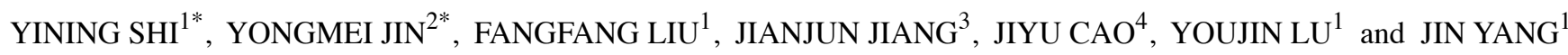 \\ ${ }^{1}$ Department of Respiratory Medicine, The Second Hospital of Anhui Medical University; \\ ${ }^{2}$ Department of Respiratory Medicine, The Second People's Hospital of Hefei; ${ }^{3}$ Department of Respiratory Medicine, \\ The First Hospital of Anhui Medical University; ${ }^{4}$ The Teaching Center for Preventive Medicine, \\ School of Public Health, Anhui Medical University, Hefei, Anhui 230022, P.R. China
}

Received December 22, 2020; Accepted March 5, 2021

DOI: $10.3892 /$ ijmm.2021.4918

\begin{abstract}
Ceramide is a biologically active sphingomyelin that inhibits cell growth and proliferation. In previous studies, it was demonstrated that the use of lipopolysaccharides induces acid sphingomyelinases to produce ceramide, promoting lung cancer cell apoptosis; however, the specific mechanisms of this action remain unclear. Thioredoxin-interacting protein (Txnip) plays an important role in the signal transmission of redox reactions inside and outside the cell. Thus, it was hypothesized that ceramide induces apoptosis in lung adenocarcinoma cells (A549 and PC9) by modulating the Txnip/Trx1 complex. In the present study, the Cell Counting kit- 8 method was used to detect cell activity and the drug concentration. Hoechst 33258 staining and flow cytometry were used to detect cell apoptosis, and the positional association between Txnip and Trx1 upregulated by ceramide was observed by immunofluorescence confocal microscopy. Reverse transcription-quantitative polymerase chain reaction and western blot analysis were used to detect the changes in related gene, mRNA and protein expression levels. The results revealed that ceramide treatment resulted in the upregulation of Txnip and in the reduction of Trx 1 activities. However, the Txnip inhibitor, verapamil, reversed these changes. The analysis of mRNA expression further verified the changes observed in the protein expression
\end{abstract}

Correspondence to: Professor Youjin Lu or Dr Jin Yang, Department of Respiratory Medicine, The Second Hospital of Anhui Medical University, 678 Furong Road, Hefei, Anhui 230022, P.R. China

E-mail: luyougold@163.com

E-mail: yangqj1015@foxmail.com

*Contributed equally

Abbreviations: Txnip, thioredoxin-interacting protein; Trx, thioredoxin; DMEM, Dulbecco's modified Eagle's medium; CCK-8, Cell Counting kit-8

Key words: ceramide, apoptosis, lung cancer, Txnip, Trx1, signaling pathway of Txnip, Trx1 and apoptosis-related proteins. On the whole, the present study demonstrates that ceramide induces the apoptosis of lung cancer cells by regulating the Txnip/Trx1 complex.

\section{Introduction}

Lung cancer is the main cause of cancer-related mortality worldwide, and non-small cell cancer accounts for approximately $85 \%$ of all lung cancer cases $(1,2)$. It is mainly characterized by a poor prognosis and a high risk of recurrence. The current treatment for lung cancer includes radiotherapy and chemotherapy. Despite the rapid development of modern technology and treatments, the mortality rate of patients with lung cancer remains high, and a tendency for relapse has been observed (3-5). Therefore, there is an urgent need to explore new drugs and treatment methods for lung cancer.

Ceramide is a biologically active sphingolipid that is the precursor of all major sphingomyelins. It plays an important role in the cell cycle, differentiation, senescence and apoptosis $(6,7)$. In addition, ceramide is considered to be an important secondary messenger in the apoptosis and necrosis pathways in normal and cancer cells (8-11). Being in the center of sphingolipid metabolism, ceramide synthesis mainly occurs via de novo, salvage and sphingomyelinase synthesis pathways. In ceramide degradation, mainly 3 ceramidases, namely alkaline, neutral and acid ceramidase, are known to be involved $(12,13)$. In previous studies, ceramide has been shown to exhibit significant antitumor activity, and its mediated antitumor activity has been reported in multiple cancer types, such as breast and prostate cancers (14-16). Inhibiting the activity of ceramidases and increasing the level of ceramide can effectively prevent resistance to chemotherapeutic drugs and induce apoptosis. Enzymes of the ceramide and ceramide synthase family play an important role in cancer $(17,18)$. However, the mechanisms through which ceramide exerts its antitumor activity remain unclear.

The thioredoxin-interacting protein (Txnip), also known as thioredoxin-binding protein 2 (TBP-2) (19), in several cases, regulates a variety of oxidative stresses together with glutathione; it can also directly inhibit the exchange between the disulfide bonds of thioredoxin (Trx)1 to inhibit the reducing 
activity of $\operatorname{Trx}$ (19-22). Trx is an important redox regulator and a sensor of energy and glucose metabolism. It is involved in various physiological processes, such as DNA damage repair, inflammation and apoptosis. It is divided into two different types, depending on the subcellular localization: Trx1 in the cytoplasm and Trx 2 in the mitochondria (23-25). Previous research has demonstrated that Trx mimetic peptides inhibit the expression of $\mathrm{p} 38 \mathrm{MAPK}$ in vitro, thereby protecting the rat brain from apoptosis and inflammation (26). Furthermore, regulating the Txnip/Trx1 complex can inhibit the activation of the NLR family pyrin domain containing 3 (NLRP3) inflammasome, thus reducing cerebral ischemia-reperfusion injury in rat (27).

Therefore, it was hypothesized that ceramide regulates the apoptosis of non-small cell lung cancer cells, induced by the Txnip/Trx1 complex. The present study aimed to examine this hypothesis by stimulating the apoptosis of non-small lung cancer cells with ceramide in vitro. In addition, the expression levels of Txnip, Trx1, p38 and other proteins in the cells detecting, and the association analyzing between Txnip and Trx1 proteins was investigated. The importance of the Txnip/Trx1 complex in ceramide-induced apoptosis of lung cancer cells was further investigated at the cellular and molecular level.

\section{Materials and methods}

Cells, reagents and antibodies. The A549 and PC9 cell lines (human lung adenocarcinoma cells) were provided by the School of Life Sciences, University of Science and Technology of China. All cells were cultured in Dulbecco's modified Eagle's medium (DMEM) (containing 10\% fetal bovine serum, $50 \mathrm{U} / \mathrm{ml}$ penicillin, and $0.05 \mathrm{mg} / \mathrm{ml}$ streptomycin) at $37^{\circ} \mathrm{C}$ in a $5 \% \mathrm{CO}_{2}$ incubator. $\mathrm{C} 2$-Ceramide and verapamil were purchased from Sigma-Aldrich; Merck KGaA. Anti- $\beta$-actin (no. ab8224), anti-p38 (no. ab170099), anti-caspase-3 (no. ab32351) and anti-cleaved caspase-3 (no. ab2302) antibodies were purchased from Abcam. Anti-Txnip (no. 18243-1-AP) antibody was purchased from ProteinTech Group. Anti-Trx (no. NBP2-52983) antibody was purchased from Novus Biologicals. The Cell Counting kit-8 (CCK-8) and apoptosis detection kits were provided by Beyotime Biotechnology, and TRIzol reagent was provided by Takara Bio, Inc.

Cell line viability determination. The CCK- 8 method was used to detect the cytotoxicity induced by $\mathrm{C} 2$-ceramide application. The A549 and PC9 cells were seeded in 96-well plates and treated with various ceramide concentration gradients of 0,20 , 50,100 and $200 \mu \mathrm{mol} / 1$. These were incubated in $37^{\circ} \mathrm{C}$ for 12 , 24 and 36 h. A microplate reader (Thermo Fisher Scientific, Inc.) was then used to detect the absorbance in each well at $450 \mathrm{~nm}$. Cell viability was calculated based on the absorbance.

Western blot analysis. Western blot analysis was used to detect the expression of Txnip, Trx1, p38, caspase-3 and cleaved-caspase-3 proteins. Cells were treated with $50 \mu \mathrm{mol} / 1$ C2-ceramide, $100 \mu \mathrm{mol} / \mathrm{l}$ verapamil and $100 \mu \mathrm{mol} / \mathrm{l}$ verapamil $+50 \mu \mathrm{mol} / 1 \mathrm{C} 2$-ceramide for $24 \mathrm{~h}$ to allow for the induction of apoptosis. The cells were then collected, lysed cells with RIPA Lysis Buffer (no. P0013K; Beyotime Institute of Biotechnology) on ice, and centrifuged at $12,000 \mathrm{x}$ g at $4^{\circ} \mathrm{C}$ for $30 \mathrm{~min}$. After measuring the protein concentration using the BCA method, the same amount of protein $(25 \mu \mathrm{g}$ per lane for each sample) was dissolved in the buffer and boiled for $10 \mathrm{~min}$ to induce denaturation. Following separation by electrophoresis on $10 \%$ SDS-PAGE, the proteins were transferred to a PVDF membrane, incubated with $5 \%$ skim milk for $1.5 \mathrm{~h}$, and then washed with water. The primary antibodies [Txnip (18243-1-AP, 1:1,000; ProteinTech Group, Inc.), Trx (NBP2-52983, 1:1,000; Novus Biologicals), p38 (ab170099, 1:2,000; Abcam), caspase-3 (ab32351, 1:2,000; Abcam) and cleaved caspase-3 (ab2302, 1:2,000; Abcam)] were applied to the membranes at room temperature for $1 \mathrm{~h}$ and the membranes were then incubated overnight at $4^{\circ} \mathrm{C}$. After washing with TBST, the corresponding secondary antibody (ab6721,1:5000; Abcam) from the same species was applied at room temperature for $1 \mathrm{~h}$ and the membranes were washed with TBST for $30 \mathrm{~min}$. Subsequently, the ECL detection kit (P0018FS; Beyotime Institute of Biotechnology) for used for signal development. Densitometry was performed by using the ImageJ software (version 1.48; National Institutes of Health).

Apoptosis detection. To further detect cell apoptosis following ceramide treatment, the cells were seeded in a 6-well plate and treated with DMEM, $50 \mu \mathrm{mol} / 1 \mathrm{C} 2$-ceramide, $50 \mu \mathrm{mol} / 1$ C2-ceramide $+100 \mu \mathrm{mol} / 1$ verapamil and $100 \mu \mathrm{mol} / 1$ verapamil for $24 \mathrm{~h}$. They were then stained with Hoechst 33258 (Beyotime Institute of Biotechnology) at room temperature for 5 min and observed under a fluorescence microscope (Olympus Corporation). Apoptotic cells were densely stained showing strong fluorescent signal in their nuclei, while non-apoptotic cells exhibited a weak fluorescence signal. In addition, cells treated in a similar manner, were labeled using fluorescein fluorescein-isothiocyanate-labeled Annexin V (C1062M; Beyotime Institute of Biotechnology) and incubated in the dark for $15 \mathrm{~min}$. The cells were then stained with propidium iodide (C1062M; Beyotime Institute of Biotechnology) for $5 \mathrm{~min}$ in the dark. The apoptotic rates of the cells were determined immediately after using flow cytometry (BD Falcon; BD Biosciences).

Caspase-3 activity detection. The treated cells were collected and washed with PBS after centrifugation. Lysis buffer (Beyotime Biotechnology) was added followed by shaking the samples for $15 \mathrm{sec}$ every $5 \mathrm{~min}$, repeated 3 times. Following centrifugation at $4^{\circ} \mathrm{C}$ and $12,000 \mathrm{~g}$ for $15 \mathrm{~min}$, the supernatant was aspirated for BCA quantification, and an appropriate amount of detection buffer was then added together with $10 \mu \mathrm{l}$ caspase- 3 substrate, and the samples were incubated at $37^{\circ} \mathrm{C}$ for 1-4 h. A microplate reader (Thermo Fisher Scientific, Inc.) was used to detect the absorbance in each well at A405 nm wavelength.

Immunofluorescence. The A549 and PC9 cells were seeded on a cover glass and treated with DMEM, $50 \mu \mathrm{mol} / 1$ C2-ceramide, $50 \mu \mathrm{mol} / 1 \mathrm{C} 2$-ceramide $+100 \mu \mathrm{mol} / 1$ verapamil, and $100 \mu \mathrm{mol} / \mathrm{l}$ verapamil for $24 \mathrm{~h}$. The cells were then fixed in $4 \%$ paraformaldehyde for $15 \mathrm{~min}$, permeabilized in $0.5 \%$ Triton X-100 for $20 \mathrm{~min}$, and then blocked in bovine serum 
A

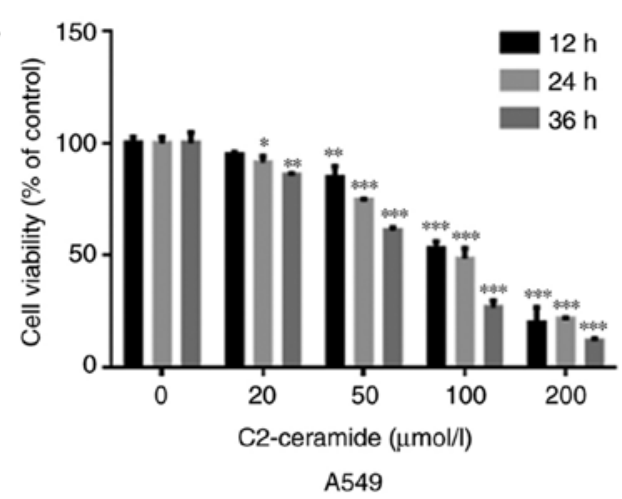

C

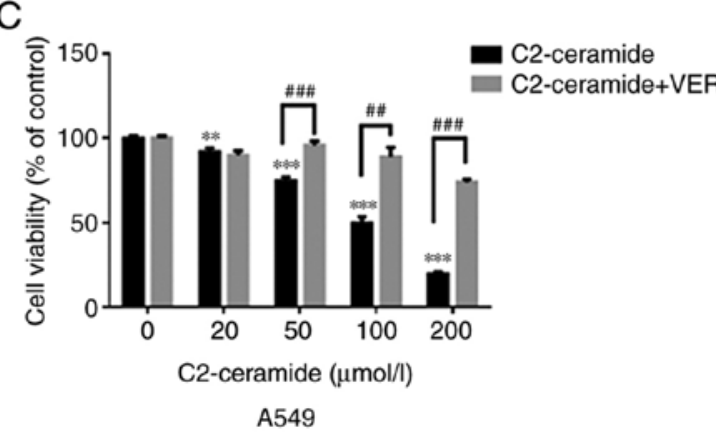

$\mathrm{B}$

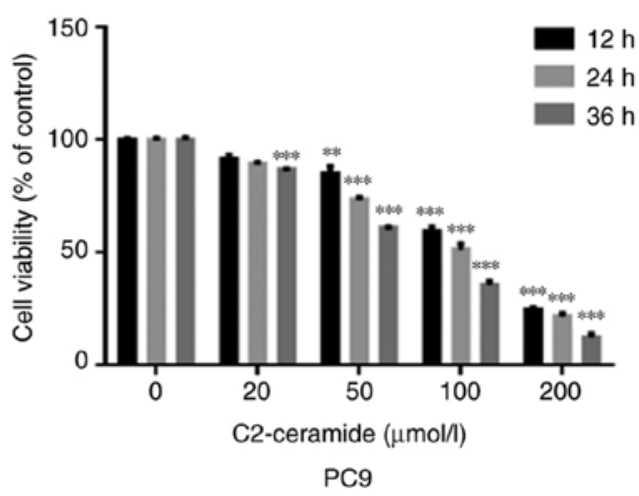

D

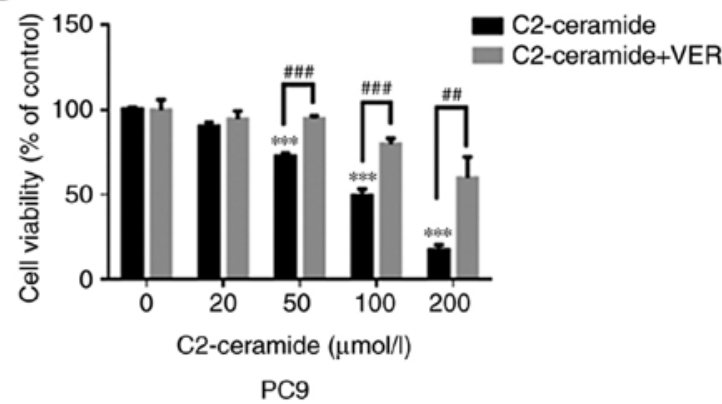

Figure 1. Effects of C2-ceramide (various concentrations and incubation times) on (A) A549 and (B) PC9 cell viability in comparison to the corresponding control group. ${ }^{*} \mathrm{P}<0.05,{ }^{* *} \mathrm{P}<0.01$ and ${ }^{* * *} \mathrm{P}<0.001$. (C) A549 and (D) PC9 cells were incubated with ceramide and verapamil for $24 \mathrm{~h}$. ${ }^{* *} \mathrm{P}<0.01$ and ${ }^{* * *} \mathrm{P}<0.001$, vs. control; ${ }^{\# \#} \mathrm{P}<0.01$ and ${ }^{\# \# \#} \mathrm{P}<0.001$, vs. ceramide.

albumin at room temperature for $30 \mathrm{~min}$. The cells were then incubated with primary antibodies to anti-Txnip (1:200; 18243-1-AP, ProteinTech Group, Inc.) and anti-Trx (1:200; NBP2-52983, Novus Biologicals) from different species at a 1:200 dilution at $4^{\circ} \mathrm{C}$ overnight. After washing with TBST, then stained with Alexa Fluor 488-labeled goat anti-mouse (1:500; SA00013-1, ProteinTech Group, Inc.) and FITC-labeled goat anti-rabbit secondary antibodies (1:500; SA00003-2, ProteinTech Group, Inc.) were added to the cells and incubated at $37^{\circ} \mathrm{C}$ in the dark for $1 \mathrm{~h}$. The cell nuclei were counterstained with DAPI (Beyotime Institute of Biotechnology) for $5 \mathrm{~min}$ at room temperature. Images were observed using a fluorescent confocal microscope (Olympus IX71, Olympus Corporation).

Reverse transcription-quantitative PCR (RT-qPCR). Total cell RNA was extracted using TRIzol reagent, and the RNA concentration was measured using a NanoDrop instrument (Thermo Fisher Scientific, Inc.). cDNA synthesis was performed using a reverse transcriptase kit (PrimeScript ${ }^{\mathrm{TM}} \mathrm{RT}$ Reagent kit -Perfect Real-Time; Takara Biotechnology, Inc.). qPCR was performed using the SYBR-Green Master Mix kit (Takara Biotechnology Co.,Ltd.) in a $10 \mu 1$ system on a real-time PCR system. The thermocycling conditions were conducted as follows: Initial denaturation $95^{\circ} \mathrm{C}$ for $5 \mathrm{~min}$, followed by 40 cycles of denaturation at $95^{\circ} \mathrm{C}$ for $15 \mathrm{sec}$, annealing at $60^{\circ} \mathrm{C}$ for $30 \mathrm{sec}$ and extension at $72^{\circ} \mathrm{C}$ for $30 \mathrm{sec}$, and a final extension at $72^{\circ} \mathrm{C}$ for $5 \mathrm{~min}$. The sequence of the primers used in the present study were as follows: Txnip forward, 5'-GCC ACACTTACCTTGCCAAT-3' and reverse, 5'-TGTTGCAGC CCAGGATAGAA-3'; Trx1 forward, 5'-GTGAAGCAGATC GAGAGCAAG-3' and reverse, 5'-CGTGGCTGAGAAGTC
AACTACTA-3'; caspase-3 forward, 5'-ATCGGACTGTGG CATTGAGA-3' and reverse, 5'-ATAACCAGGTGCTGTGGA GT-3'; and $\beta$-actin forward, 5'-CCCTGGAGAAGAGCTACG AG-3' and reverse, 5'-GGAAGGAAGGCTGGAAGAGT-3'. The expression of each target gene was statistically compared with the RNA level using the $2^{-\Delta \Delta C q}$ method (28), and $\beta$-actin was used as an internal reference.

Statistical analysis. All experiments were repeated 3 times. Statistical analysis was performed using SPSS 21.0 (IBM Corp). All data are expressed as the means $+\mathrm{SD}$, and the data were analyzed using the Student's t-test or one-way ANOVA followed by post hoc comparisons with Student-Newman-Keuls (S) and Tukey's post hoc tests. $\mathrm{P}<0.05$ was considered to indicate a statistically significant difference.

\section{Results}

Effect of ceramide on cell viability. As shown in Fig. 1A (A549 cells) and B (PC9 cells), ceramide reduced cell viability in a time- and concentration-dependent manner. The cell survival rate in the treatment group was significantly lower than that in the control group when the ceramide concentration was 50, 100 and $200 \mu \mathrm{mol} / 1(\mathrm{P}<0.05)$. When the concentration of the treatment group was $50 \mu \mathrm{mol} / 1$ and the treatment duration was $24 \mathrm{~h}$, the cell viability in the 2 treatment groups was approximately $70 \%$. However, when verapamil was incubated with C2-ceramide, the cell viability was increased (Fig. 1C and D).

Apoptosis promoted by ceramide is affected by verapamil. As shown in Fig. 2, the results of Hoechst 33258 staining revealed 
A
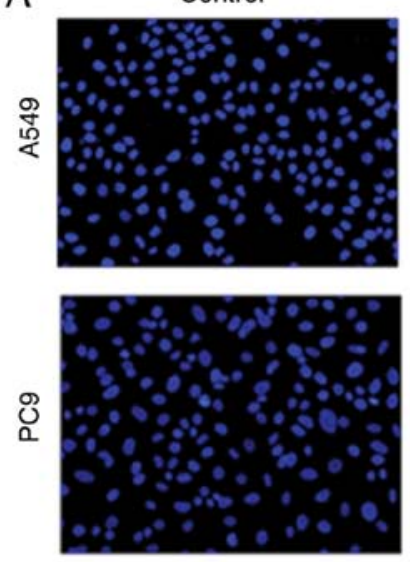

C2-ceramide
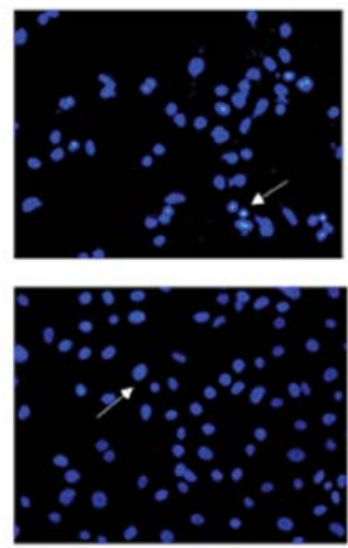

C2-ceramide+VER
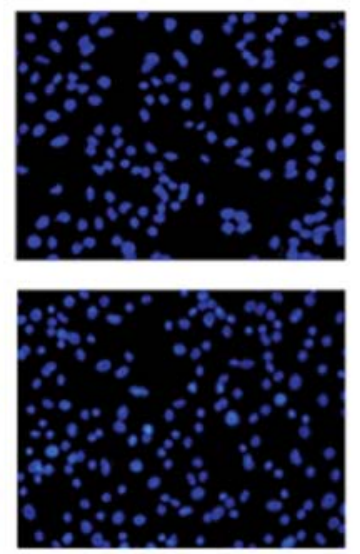

VER
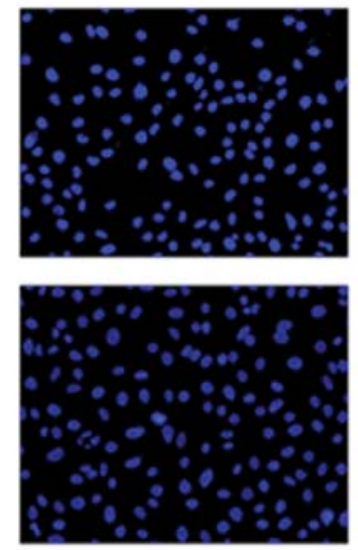
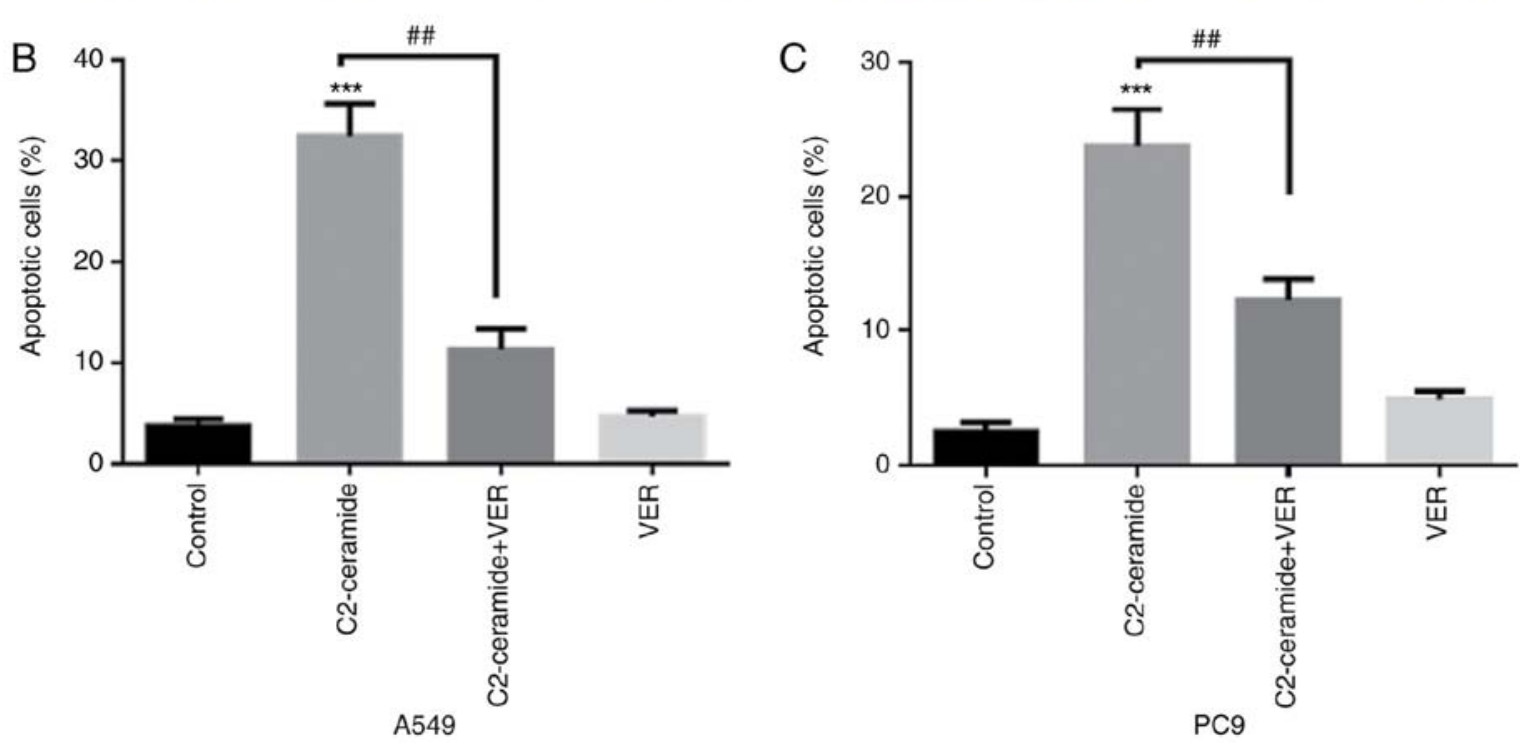

Figure 2. Hoechst 33258 is used to detect the effects of C2-ceramide (50 $\mu \mathrm{mol} / \mathrm{l})$ and verapamil (VER, $100 \mu \mathrm{mol} / \mathrm{l})$ treatment in (A and B) A549 and (A and C) PC9 cell lines following $24 \mathrm{~h}$ of incubation. ${ }^{* * *} \mathrm{P}<0.001$, vs. control; ${ }^{\# \#} \mathrm{P}<0.01$ vs. ceramide. Images are captured from random fields of view at a magnification of $\mathrm{x} 200$, Scale bar, $50 \mu \mathrm{m}$.

that the apoptotic rates of the two cell lines were significantly higher in the ceramide-treated group than in the control group. Moreover, the use of verapamil significantly reduced the number of cells undergoing apoptosis $(\mathrm{P}<0.05)$. The results of flow cytometry revealed shown in Fig. 3 also demonstrated similar trends $(\mathrm{P}<0.05)$. Furthermore, caspase- 3 activity was enhanced in the ceramide group, and co-incubation with verapamil rescued the cells from apoptosis $(\mathrm{P}<0.05)$, as shown in Fig. 4. The expression of apoptosis-related proteins also exhibited a similar tendency (Fig. 5).

Effect of ceramide on Txnip and Trxl on the promotion of apoptosis. The results of immunofluorescence staining shown in Fig. 6A (A549 cells) and B (PC9 cells) revealed that compared to the control group, the two cell lines exhibited a significantly increased fluorescence signal corresponding to the expression of Txnip in the cytoplasm. Txnip is typically located in the nucleus, and Txnip translocation occurred in response to ceramide treatment. This indicates that Txnip reacts through the cell membrane during apoptosis. Following co-incubation with verapamil, the expression of Txnip in the cytoplasm decreased. This trend was inversely related to the expression of Trx1. The protein expression level also exhibited similar results, as shown in Fig. 5.

Expression of Txnip, Trxl and caspase-3 at the mRNA level. As shown in Fig. 7, compared to the control group, the Txnip expression level (Fig. 7A and D) was significantly increased after the two cell lines were treated with $50 \mu \mathrm{mol} / 1 \mathrm{C} 2$-ceramide, and the Trx1 level (Fig. 7B and E) was significantly decreased $(\mathrm{P}<0.05)$. Compared to the control group, the expression levels of apoptotic proteins were also increased (Fig. 7C and F). Following co-incubation with $100 \mu \mathrm{mol} / 1$ verapamil, this pattern of expression was significantly reversed $(\mathrm{P}<0.05)$.

\section{Discussion}

Studies have demonstrated that ceramide is closely related to apoptosis and cancer. Ceramide inducers can enhance the sensitivity to anticancer drugs $(7,12,29)$. In previous studies $(30,31)$, the authors demonstrated that ceramide was significantly increased in in mice with lipopolysaccharide-induced ALI, and the survival rate in mice was reduced. The use of the aSmase inhibitor, imipramine, reduced lung injury. However, the specific pathway 

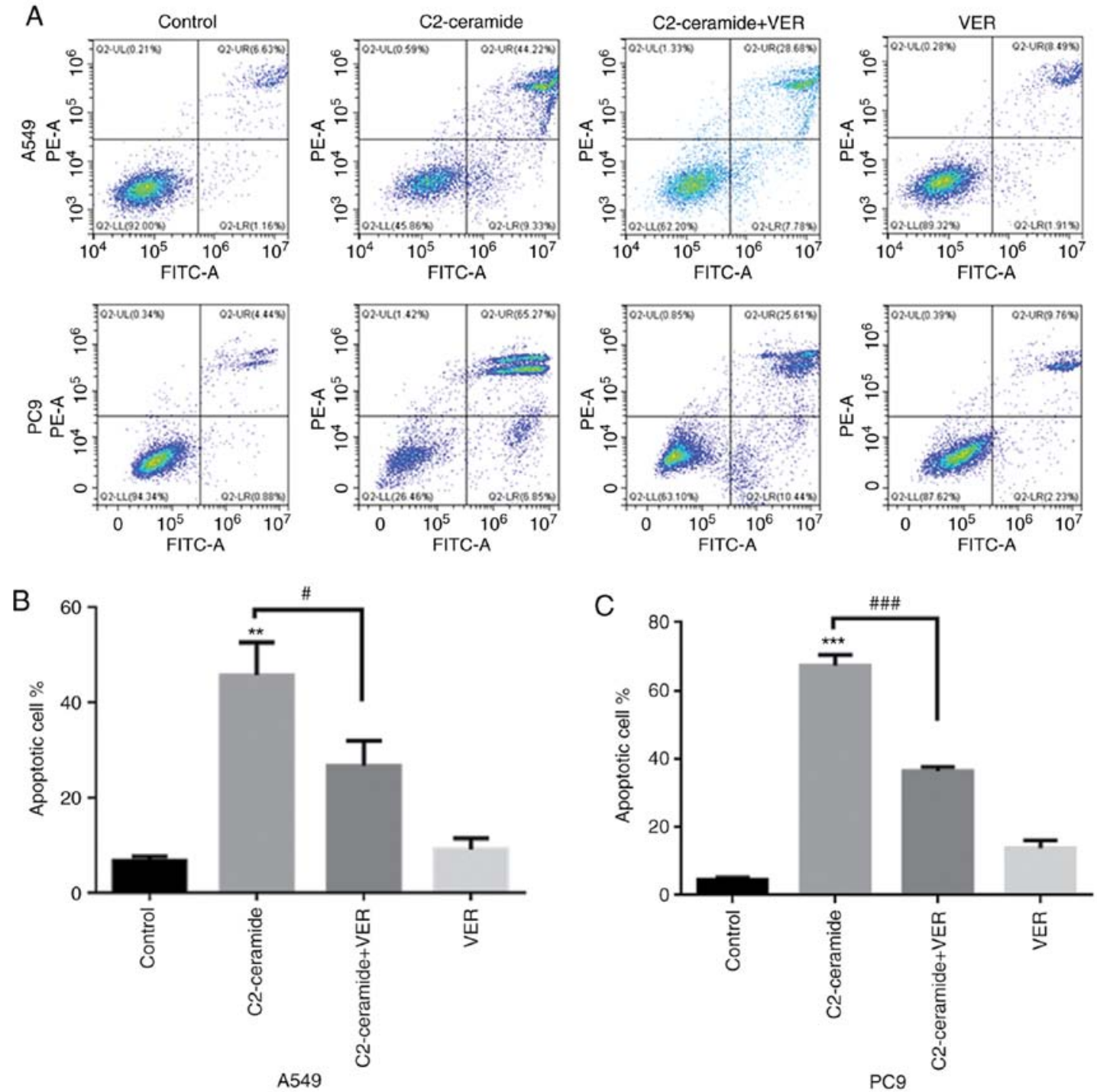

Figure 3. Flow cytometry detected the effects of C2-ceramide ( $50 \mu \mathrm{mol} / 1)$ and $100 \mu \mathrm{mol} / 1$ verapamil treatment in (A and B) A549 and (A and C) PC9 cell lines following $24 \mathrm{~h}$ of treatment, ${ }^{* *} \mathrm{P}<0.01$ and ${ }^{* * *} \mathrm{P}<0.001$, vs. control; ${ }^{\#} \mathrm{P}<0.05$ and ${ }^{\# \# \#} \mathrm{P}<0.001$, vs. ceramide.

A

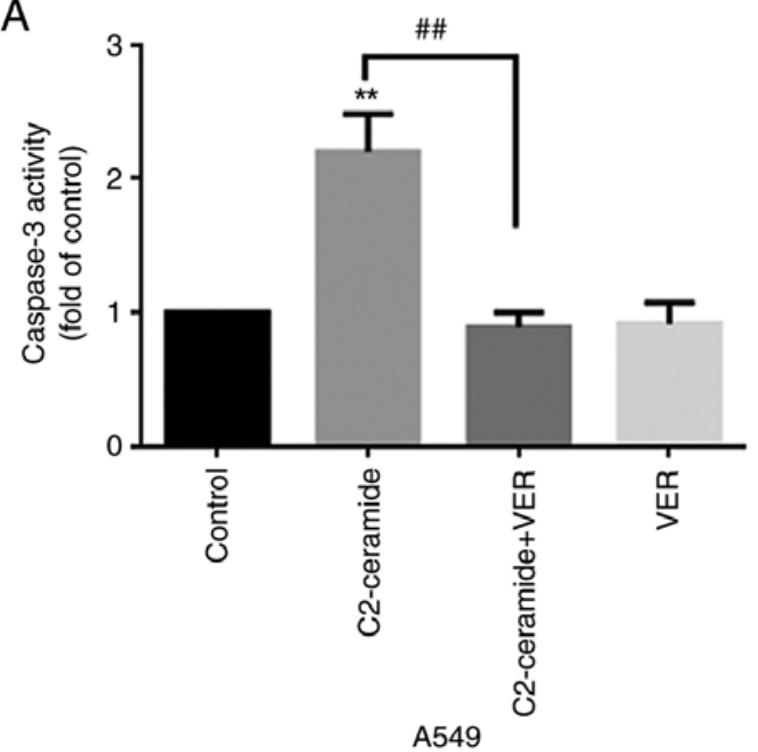

B

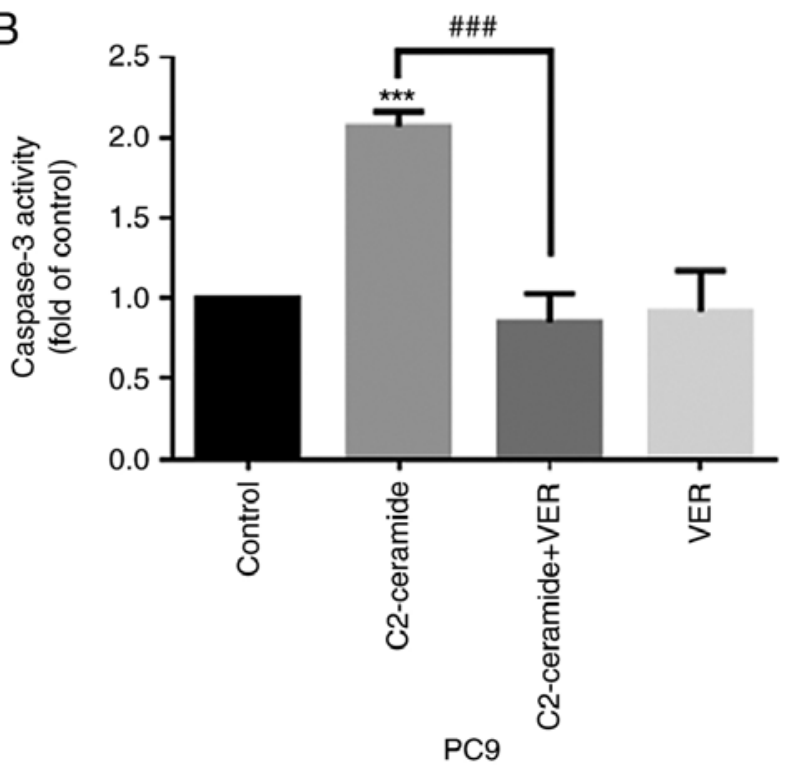

Figure 4. Caspase-3 activity is detected after C2-ceramide-induced apoptosis of (A) A549 and (B) PC9 cells, compared with the control group. The activity of caspase-3 in the $\mathrm{C} 2$-ceramide treatment group was increased. ${ }^{* *} \mathrm{P}<0.01$ and ${ }^{* * *} \mathrm{P}<0.001$, vs. control; ${ }^{\# \#} \mathrm{P}<0.01$ and ${ }^{\# \# \#} \mathrm{P}<0.001$, vs. ceramide. 

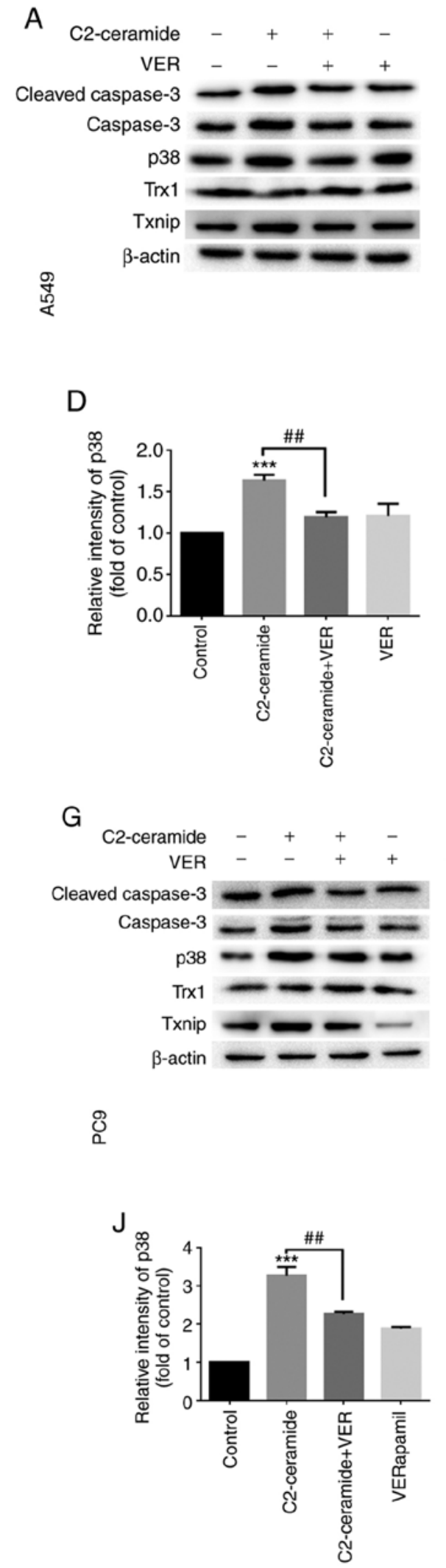

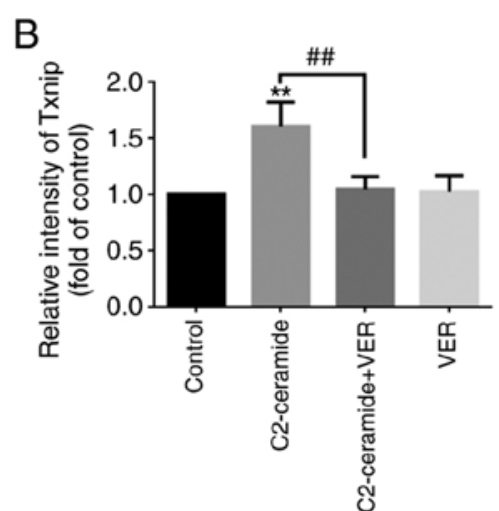

E
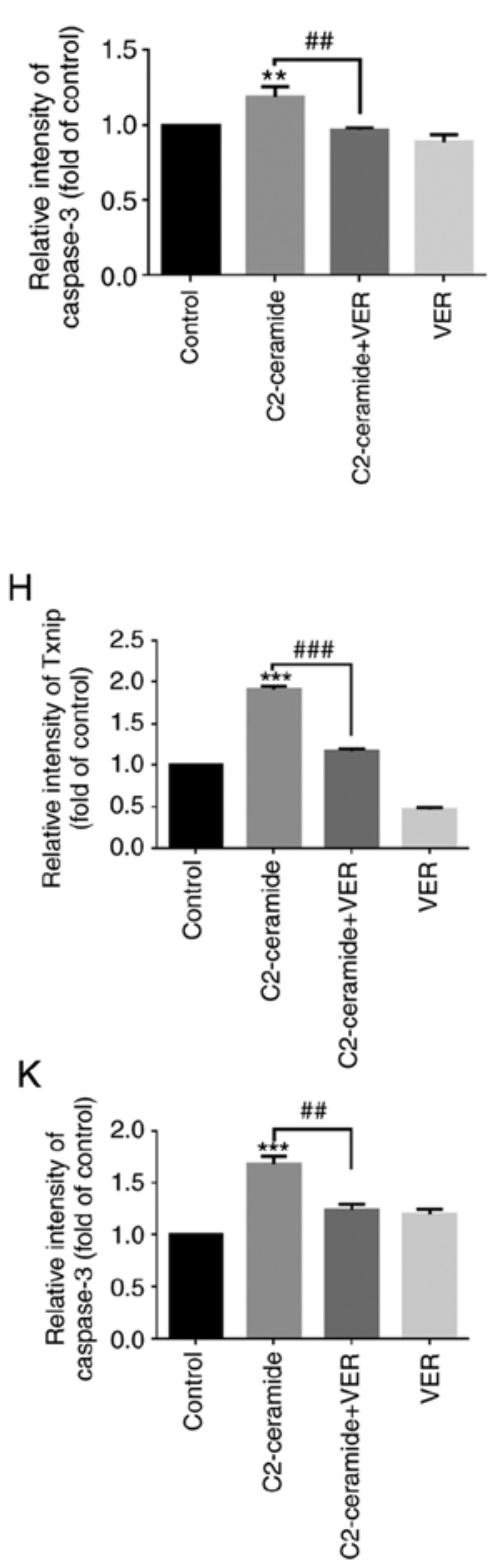
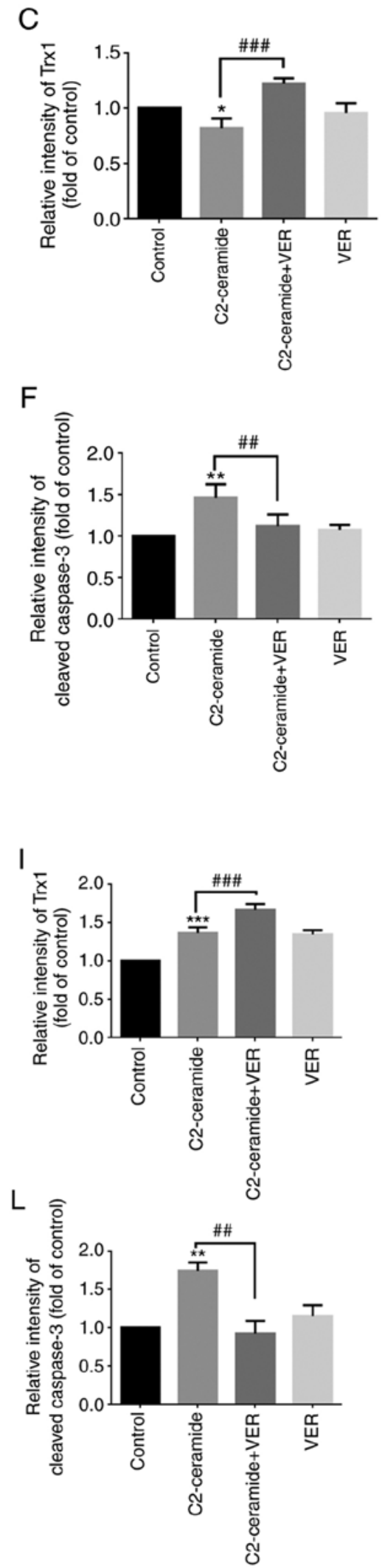

Figure 5. C2-ceramide promotes apoptosis-related protein expression, thereby enhancing (A-F) A549 and (G-L) PC9 cell apoptosis as compared to the corresponding control group. The expression of $\beta$-actin was used as an internal control. ${ }^{*} \mathrm{P}<0.05,{ }^{* *} \mathrm{P}<0.01$ and ${ }^{* * * *} \mathrm{P}<0.001$, vs. control; ${ }^{* \#} \mathrm{P}<0.01$ and

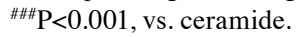

through which ceramide induces lung cancer cell apoptosis is not yet fully understood. In the pre-experiment before this research (data not shown), the H1299, H1975, A549 and PC9 cell lines were tested and it was found that the A549 and PC9 cells had the most significant drug response to ceramide treatment. Therefore, in the present study, the A549 and PC9 cell lines were used in the 
A
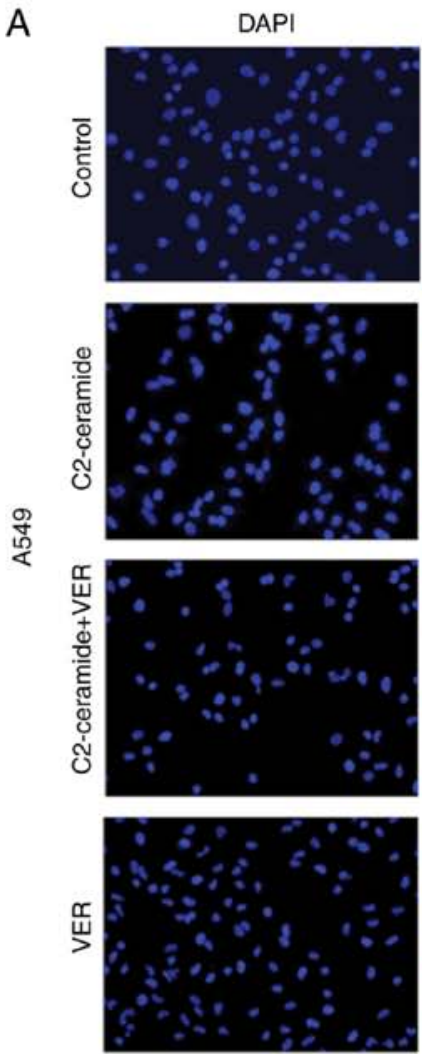

B
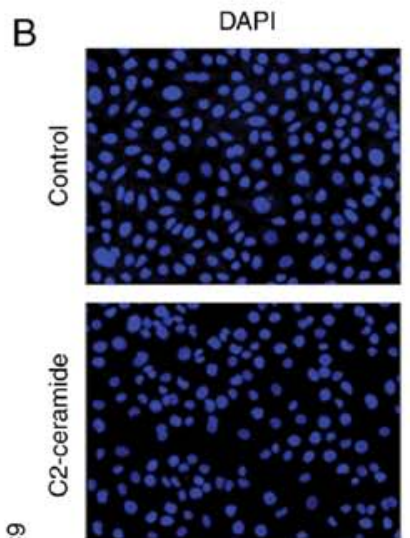

임

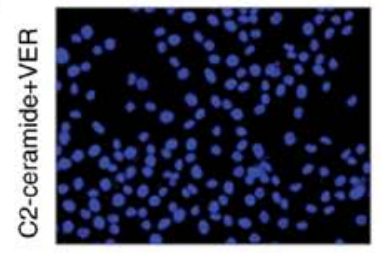

㐍

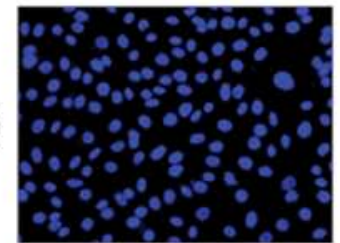

Txnip
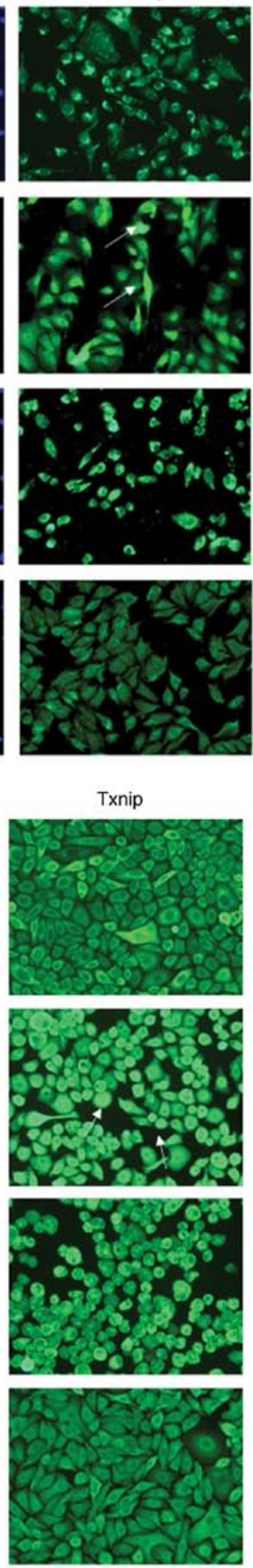

Trx1
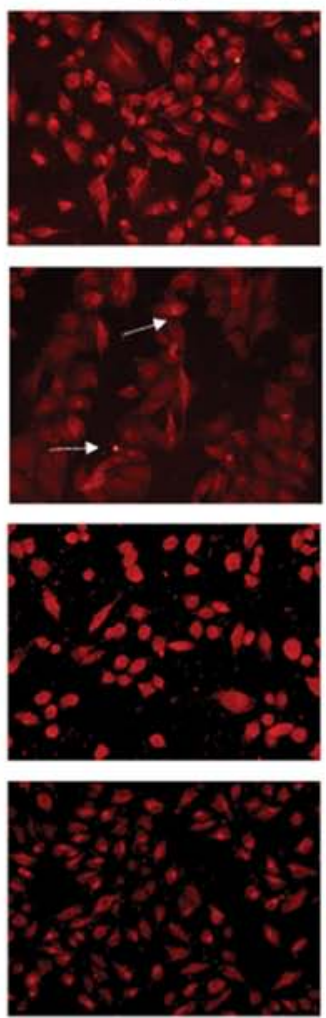

Trx1
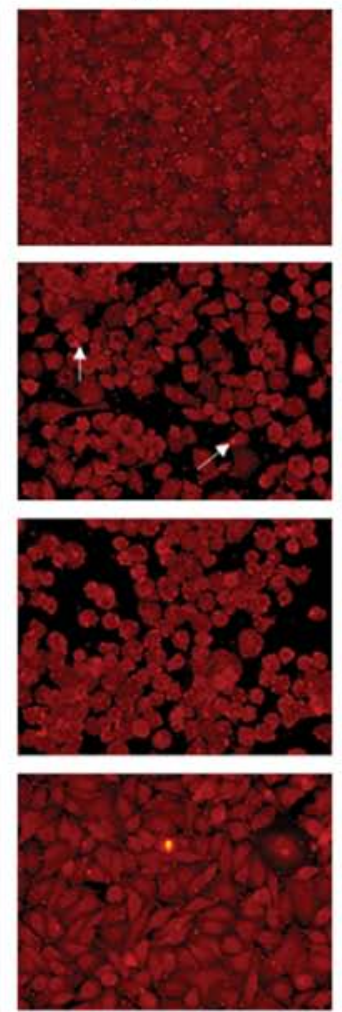
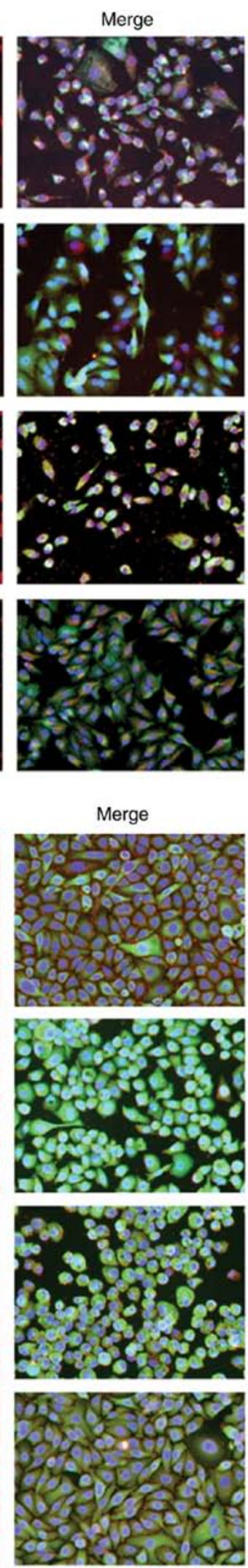

Figure 6. Immunofluorescence staining showing that compared to the control group, ceramide treatment significantly enhanced the fluorescence intensity in Txnip in (A) A549 and (B) PC9 cells after 24 h, while it reduced Trx1 fluorescence intensity. Co-incubation with verapamil reversed this expression pattern. The interaction of Txnip with Trx1 was determined using a confocal microscopic observation. Images were captured from random fields of view at a magnification of $\mathrm{x} 200$, Scale bar, $50 \mu \mathrm{m}$.

experiments and it was proven that ceramide induced non-small cell lung cancer apoptosis through the Txnip/Trx1 complex.
Lung cancer is the main cause of cancer-related mortality worldwide, and the average 5-year survival rate is only 

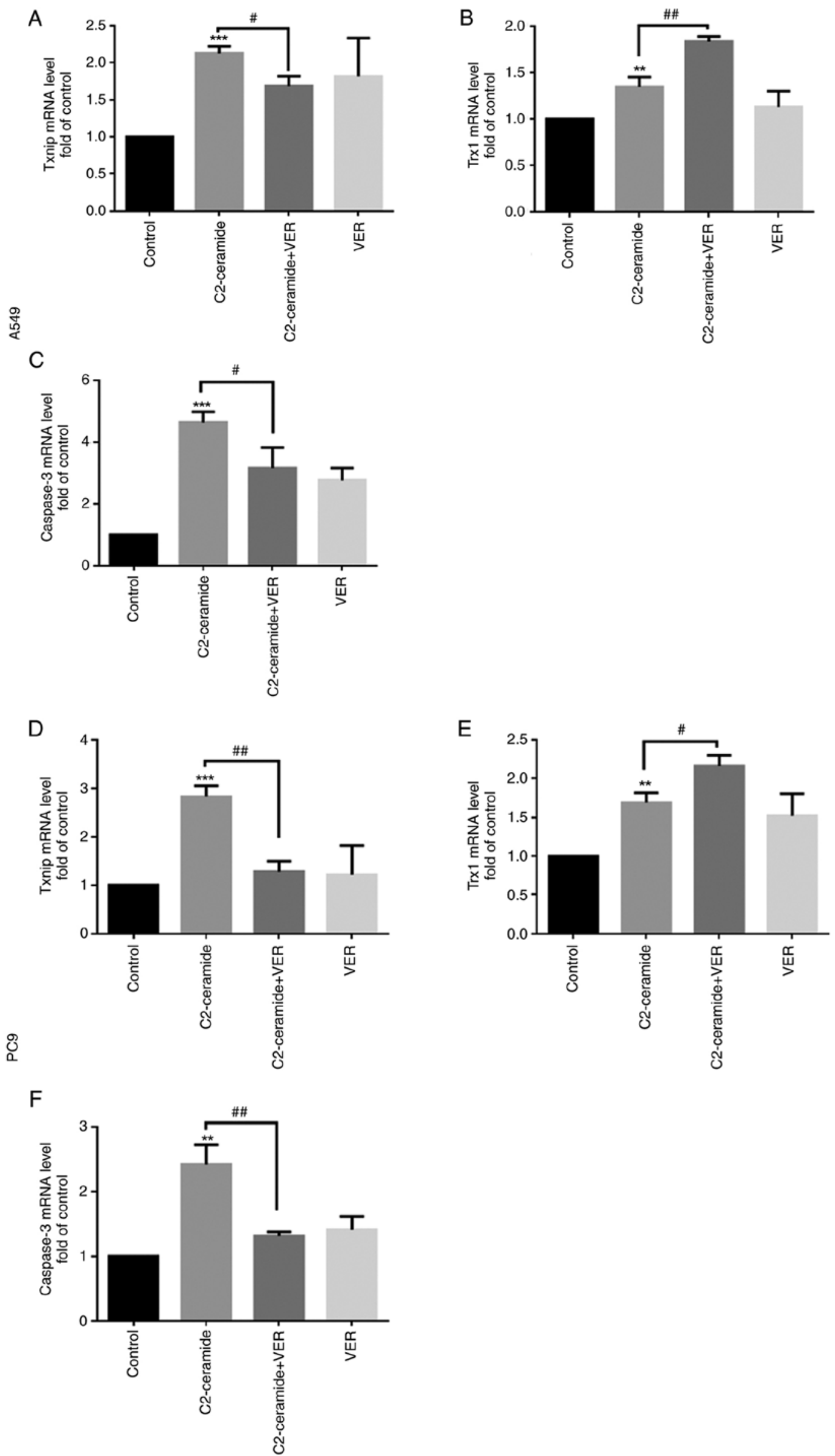

Figure 7. C2-ceramide promotes corresponding mRNA expression, thereby enhancing (A-C) A549 and (D-F) PC9 cell apoptosis as compared to the corresponding control group. ${ }^{* *} \mathrm{P}<0.01$ and ${ }^{* * *} \mathrm{P}<0.001$, vs. control; ${ }^{*} \mathrm{P}<0.05$, and ${ }^{\# \#} \mathrm{P}<0.01$, vs. ceramide. 
$19 \%(32,33)$. Although chemotherapy is the main treatment method, its side-effects pose a significant challenge. Therefore, alternative chemotherapeutic drugs and those that reduce the side-effects of chemotherapy are required (34). Studies have demonstrated that sphingolipid metabolism-targeted therapy may have broad prospects in oncological clinical application. The regulatory effects of ceramide on chemotherapeutics have become the focus of research in recent years $(35,36)$. The human lung adenocarcinoma cell lines, A549 and PC9, are non-small cell lung cancer cell lines. The present study revealed that ceramide reduced cell viability in a time- and concentration-dependent manner. Following treatment with $50 \mu \mathrm{mol} / 1$ ceramide, caspase-3 activity increased, and the levels of apoptosis-related proteins, such as Txnip and Trx1 were also altered accordingly. This indicated that Txnip and Trx 1 were downstream of ceramide during the induction of cell apoptosis.

Txnip is a tumor suppressor gene and an endogenous inhibitor of Trx. Usually, Txnip binds to Trx1 in a redox-dependent manner and participates in various redox reactions $(27,37,38)$. Previous research has demonstrated that the activation of the Txnip/Trx1 complex increases oxidative stress and inflammation, thereby aggravating the symptoms of diabetes; this finding proved that the upregulation of Txnip or activation of the Txnip/Trx 1 complex plays an important role in aggravating diabetic myocardial damage (39). The present study also demonstrated, by immunofluorescence staining, that Txnip induced by C2-ceramide co-localized with Trx1. Txnip is mainly localized in the nucleus, while Trx1 is mainly distributed in the cytoplasm. The fluorescent expression of Txnip was significantly increased, while Trx 1 expression was reduced. Verapamil treatment reversed this expression-dose association. The decreased Trx 1 activity was mainly caused by the increase in Txnip; however, other factors that can influence this process may be involved as well (19). It has been demonstrated that radiation, hydrogen peroxide, ultraviolet light and growth inhibition stimulates Txnip production (40-42). p38 MAPK is a protein kinase that responds to stress stimuli inside and outside the cell. It plays an important role in regulating cell differentiation, proliferation, survival and death. Studies have proven the central role of p38 MAPK in inflammation $(43,44)$. In the present study, when verapamil was used to inhibit ceramide-induced Txnip expression, the expression of $\mathrm{p} 38$ protein was decreased. It can thus be inferred that the change in p38 protein expression was affected by Txnip protein, which acts upstream of p38. However, further experiments are required to confirm the accuracy of this interpretation.

In conclusion, the present study investigated the anticancer mechanisms of sphingolipid ceramide action. It was found that it induced lung cancer cell apoptosis through the Txnip/Trx1 complex, which plays an important role in biological activities, such as oxidative stress. Ceramide has great potential and is expected to become a prospective candidate in the development of therapies for cancer. In addition, research on various targets in the Txnip/Trx1 complex may provide new possibilities for cancer treatment. However, the present study also had limitations. First, the present study was limited to the experiments performed at the cellular level in vitro. The human body is more complex and numerous factors are involved. Thus, the same effect of ceramide on the human body as that observed in cell culture cannot be guaranteed. Second, the present study selected only one representative type of ceramide. Differences across the other types of ceramide are unclear. Furthermore, only used two types of lung adenocarcinoma cells were used. The effect of ceramide on other types of lung cancer cells thus warrant further investigation.

\section{Acknowledgements}

Not applicable.

\section{Funding}

The present study was supported by the National Natural Science Foundation of China (grant no. 81400058) and the Anhui Province Science and Technology Tackling Plan Project (no. 1401045016).

\section{Availability of data and materials}

All data generated or analyzed during this study are included in this published article.

\section{Authors' contributions}

YS and YJ designed the study, performed the immunofluorescence assay and drafted the manuscript. JJ and FL designed the study, performed the cell viability assays and analyzed the data. YS was responsible for cell culture. YS and YJ performed the western blot analysis. YL, JY and JC were involved in the study design, quality control, manuscript drafting and coordination. YS and YJ confirm the authenticity of all the raw data. All authors have read and approved the final draft.

\section{Ethics approval and consent to participate}

Not applicable.

\section{Patient consent for publication}

Not applicable.

\section{Competing interests}

The authors declare that they have no competing interests.

\section{References}

1. Remark R, Becker C, Gomez JE, Damotte D, Dieu-Nosjean MC, Sautès-Fridman C, Fridman WH, Powell CA, Altorki NK, Merad M and Gnjatic S: The non-small cell lung cancer immune contexture. A major determinant of tumor characteristics and patient outcome. Am J Respir Crit Care Med 191: 377-390, 2015.

2. Skřičková J, Kadlec B, Venclíček O and Merta Z: Lung cancer. Cas Lek Cesk 157: 226-236, 2018.

3. Misra P and Singh S: Role of cytokines in combinatorial immunotherapeutics of non-small cell lung cancer through systems perspective. Cancer Med 8: 1976-1995, 2019.

4. Naylor EC, Desani JK and Chung PK: Targeted therapy and immunotherapy for lung cancer. Surg Oncol Clin N Am 25: 601-609, 2016.

5. Owen DH, Williams TM, BertinoEM, MoX, Webb A, Schweitzer C, Liu T, Roychowdhury S, Timmers CD and Otterson GA: Homologous recombination and DNA repair mutations in patients treated with carboplatin and nab-paclitaxel for metastatic non-small cell lung cancer. Lung Cancer 134: 167-173, 2019. 
6. Kus G, Özkurt M, Öztopcu Vatan P, Erkasap N, Uyar R and Kabadere S: Comparison of a ceramidase inhibitor (ceranib-2) with $\mathrm{C} 2$ ceramide and cisplatin on cytotoxicity and apoptosis of glioma cells. Turk J Biol 42: 259-265, 2018.

7. Ordoñez R, Fernández A, Prieto-Domínguez N, Martínez L, García-Ruiz C, Fernández-Checa JC, Mauriz JL and González-Gallego J: Ceramide metabolism regulates autophagy and apoptotic cell death induced by melatonin in liver cancer cells. J Pineal Res 59: 178-189, 2015.

8. Kolesnick R and Fuks Z: Radiation and ceramide-induced apoptosis. Oncogene 22: 5897-5906, 2003.

9. Brodowicz J, Przegaliński E, Müller CP and Filip M: Ceramide and its related neurochemical networks as targets for some brain disorder therapies. Neurotox Res 33: 474-484, 2018

10. Chang YC, Fong Y, Tsai EM, Chang YG, Chou HL, Wu CY, Teng YN, Liu TC, Yuan SS and Chiu CC: Exogenous $\mathrm{C}_{8}$-ceramide induces apoptosis by overproduction of ROS and the switch of superoxide dismutases SOD1 to SOD2 in human lung cancer cells. Int J Mol Sci 19: 3010, 2018.

11. Ma JQ, Liu CM and Yang W: Protective effect of rutin against carbon tetrachloride-induced oxidative stress, inflammation and apoptosis in mouse kidney associated with the ceramide, MAPKs, p53 and calpain activities. Chem Biol Interact 286: 26-33, 2018.

12. Maeng HJ, Song JH, Kim GT, Song YJ, Lee K, Kim JY and Park TS: Celecoxib-mediated activation of endoplasmic reticulum stress induces de novo ceramide biosynthesis and apoptosis in hepatoma HepG2 cells mobilization. BMB Rep 50: 144-149, 2017.

13. Wang SW, Hojabrpour P, Zhang P, Kolesnick RN, Steinbrecher UP, Gómez-Muñoz A and Duronio V: Regulation of ceramide generation during macrophage apoptosis by ASMase and de novo synthesis. Biochim Biophys Acta 1851: 1482-1489, 2015.

14. Dai L, Smith CD, Foroozesh M, Miele L and Qin Z: The sphingosine kinase 2 inhibitor ABC294640 displays anti-non-small cell lung cancer activities in vitro and in vivo. Int J Cancer 142 2153-2162, 2018.

15. Chang WT, Wu CY, Lin YC, Wu MT, Su KL, Yuan SS, Wang HD, Fong Y, Lin YH and Chiu CC: C(2)-ceramide-induced $\mathrm{Rb}$-dominant senescence-like phenotype leads to human breast cancer MCF-7 escape from p53-dependent cell death. Int J Mol Sci 20: 4292, 2019.

16. Rahman A, Pallichankandy S, Thayyullathil $\mathrm{F}$ and Galadari $\mathrm{S}$ : Critical role of $\mathrm{H}(2) \mathrm{O}(2)$ in mediating sanguinarine-induced apoptosis in prostate cancer cells via facilitating ceramide generation, ERK1/2 phosphorylation, and Par-4 cleavage. Free Radic Biol Med 134: 527-544, 2019.

17. Yildiz-Ozer M, Oztopcu-Vatan P and Kus G: The investigation of ceranib-2 on apoptosis and drug interaction with carboplatin in human non small cell lung cancer cells in vitro. Cytotechnology 70: 387-396, 2018.

18. Suzuki M, Cao K, Kato S, Komizu Y, Mizutani N, Tanaka K, Arima C, Tai MC, Yanagisawa K, Togawa N, et al: Targeting ceramide synthase 6-dependent metastasis-prone phenotype in lung cancer cells. J Clin Invest 126: 254-265, 2016.

19. Xu L, Lin X, Guan M, Zeng Y and Liu Y: Verapamil attenuated prediabetic neuropathy in high-fat diet-fed mice through inhibiting TXNIP-mediated apoptosis and inflammation. Oxid Med Cell Longev 2019: 1896041,2019.

20. Kizhakkayil J, Thayyullathil F, Chathoth S, Hago A, Patel M and Galadari S: Glutathione regulates caspase-dependent ceramide production and curcumin-induced apoptosis in human leukemic cells. Free Radic Biol Med 52: 1854-1864, 2012.

21. Xu W, Wang L, Li J, Cai Y and Xue Y: TXNIP mediated the oxidative stress response in glomerular mesangial cells partially through AMPK pathway. Biomed Pharmacother 107: 785-792, 2018.

22. Lv H, Zhu C, Wei W, Lv X, Yu Q, Deng X and Ci X: Enhanced Keap1-Nrf2/Trx-1 axis by daphnetin protects against oxidative stress-driven hepatotoxicity via inhibiting ASK1/JNK and Txnip/NLRP3 inflammasome activation. Phytomedicine 71: $153241,2020$.

23. Yu Y, Xing K, Badamas R, Kuszynski CA, Wu H and Lou MF: Overexpression of thioredoxin-binding protein 2 increases oxidation sensitivity and apoptosis in human lens epithelial cells. Free Radic Biol Med 57: 92-104, 2013.

24. Muri J, Heer S, Matsushita M, Pohlmeier L, Tortola L, Fuhrer T, Conrad M, Zamboni N, Kisielow J and Kopf M: The thioredoxin-1 system is essential for fueling DNA synthesis during T-cell metabolic reprogramming and proliferation. Nat Commun 9: 1851, 2018.
25. Ungerstedt J, Du Y, Zhang H, Nair D and Holmgren A: In vivo redox state of human thioredoxin and redox shift by the histone deacetylase inhibitor suberoylanilide hydroxamic acid (SAHA). Free Radic Biol Med 53: 2002-2007, 2012.

26. Cohen-Kutner M, Khomsky L, Trus M, Ben-Yehuda H, Lenhard JM,Liang Y, Martin T and Atlas D: Thioredoxin-mimetic peptide CB3 lowers MAPKinase activity in the Zucker rat brain. Redox Biol 2: 447-456, 2014.

27. Hou Y, Wang Y, He Q, Li L, Xie H, Zhao Y and Zhao J: Nrf2 inhibits NLRP3 inflammasome activation through regulating Trx1/TXNIP complex in cerebral ischemia reperfusion injury. Behav Brain Res 336: 32-39, 2018.

28. Livak KJ and Schmittgen TD: Analysis of relative gene expression data using real-time quantitative PCR and the 2(-Delta Delta C(T)) method. Methods 25: 402-408, 2001

29. Morad SA, Davis TS, MacDougall MR, Tan SF, Feith DJ, Desai DH, Amin SG, Kester M, Loughran TP Jr and Cabot MC: Role of P-glycoprotein inhibitors in ceramide-based therapeutics for treatment of cancer. Biochem Pharmacol 130: 21-33, 2017.

30. Liu H, Yang J, Wang Y, Wei Y, Cao J and Lu Y: C8-ceramide induces apoptosis of alveolar type II epithelial cells. Chinese J Tuberculosis Respir Zhonghua Jie He He Hu Xi Za Zhi 38: 445-450, 2015 (In Chinese).

31. Yang J, Wang Y, Liu H, Bi J and Lu Y: C2-ceramide influences alveolar epithelial barrier function by downregulating $\mathrm{Zo}_{\mathrm{o}} 1$, occludin and claudin-4 expression. Toxicol Mech Methods 27: 293-297, 2017.

32. Siegel RL, Miller KD and Jemal A: Cancer statistics, 2019. CA Cancer J Clin 69: 7-34, 2019.

33. Mattiuzzi $\mathrm{C}$ and Lippi G: Current cancer epidemiology. J Epidemiol Glob Health 9: 217-222, 2019.

34. Du A, Jiang Y and Fan C: NDRG1 downregulates ATF3 and inhibits cisplatin-induced cytotoxicity in lung cancer A549 cells. Int J Med Sci 15: 1502-1507, 2018.

35. Ogretmen B: Sphingolipid metabolism in cancer signalling and therapy. Nat Rev Cancer 18: 33-50, 2018.

36. Che J, Huang Y, Xu C and Zhang P: Increased ceramide production sensitizes breast cancer cell response to chemotherapy. Cancer Chemother Pharmacol 79: 933-941, 2017

37. World C, Spindel ON and Berk BC: Thioredoxin-interacting protein mediates TRX1 translocation to the plasma membrane in response to tumor necrosis factor- $\alpha$ : A key mechanism for vascular endothelial growth factor receptor- 2 transactivation by reactive oxygen species. Arterioscler Thromb Vasc Biol 31: 1890-1897, 2011

38. Bharti V, Tan H, Zhou H and Wang JF: Txnip mediates glucocorticoid-activated NLRP3 inflammatory signaling in mouse microglia. Neurochem Int 131: 104564, 2019.

39. Hou R, Shen M, Wang R, Liu H, Gao C, Xu J, Tao L, Yin Z and Yin T: Thioredoxin1 inactivation mediates the impairment of ischemia-induced angiogenesis and further injury in diabetic myocardium. J Vasc Res 57: 76-85, 2020.

40. Yoshihara E: TXNIP/TBP-2: A master regulator for glucose homeostasis. Antioxidants (Basel) 9: 765, 2020.

41. Nishiyama A, Matsui M, Iwata S, Hirota K, Masutani H, Nakamura H, Takagi Y, Sono H, Gon Y and Yodoi J: Identification of thioredoxin-binding protein-2/vitamin $D(3)$ up-regulated protein 1 as a negative regulator of thioredoxin function and expression. J Biol Chem 274: 21645-21650, 1999.

42. Wang Y, De Keulenaer GW and Lee RT: Vitamin $\mathrm{D}$ (3)-up-regulated protein-1 is a stress-responsive gene that regulates cardiomyocyte viability through interaction with thioredoxin. J Biol Chem 277: 26496-26500, 2002.

43. Li D, Ren W, Jiang Z and Zhu L: Regulation of the NLRP3 inflammasome and macrophage pyroptosis by the p38 MAPK signaling pathway in a mouse model of acute lung injury. Mol Med Rep 18: 4399-4409, 2018.

44. Song W, Wei L, Du Y, Wang Y and Jiang S: Protective effect of ginsenoside metabolite compound $\mathrm{K}$ against diabetic nephropathy by inhibiting NLRP3 inflammasome activation and NF- $\kappa \mathrm{B} / \mathrm{p} 38$ signaling pathway in high-fat diet/streptozotocin-induced diabetic mice. Int Immunopharmacol 63: 227-238, 2018.

This work is licensed under a Creative Commons Attribution-NonCommercial-NoDerivatives 4.0 International (CC BY-NC-ND 4.0) License. 